\title{
One-point multipactor in crossed fields of rf cavities
}

\author{
Valery Shemelin* \\ Cornell Laboratory for Accelerator-based Sciences and Education (CLASSE), Cornell University, Ithaca, New York 14853, USA
}

(Received 23 August 2013; published 29 October 2013)

\begin{abstract}
This is a complementary paper to the recently published paper on the two-point multipactor (MP2) in a superconducting cavity near its equator [V. Shemelin, Phys. Rev. ST Accel. Beams 16, 012002 (2013)]. Now, the analytical description of the one-point multipactor (MP1) is suggested following the approach used for MP2. Such a description gives a clear physical understanding of many features of the phenomenon. Influence of the cavity shape on the existence of this or another kind of the discharge is analyzed. A concept of a geometrical parameter of multipactor is introduced. This parameter is different for different kinds of MP and defines limits of its existence.
\end{abstract}

DOI: 10.1103/PhysRevSTAB.16.102003

\section{INTRODUCTION}

Electron multipacting is a well-known phenomenon that can be met in many radio frequency (rf) devices. It is especially important to avoid this kind of discharge in the superconducting (SC) rf cavities when their best advantage, low losses, is compromised by the avalanche increase of the electrons bombarding the cavity walls which can lead to the loss of superconductivity.

One-point and two-point multipacting are distinguished in the rf devices, however no clear physical explanations why this or another kind of the discharge exists in an rf device were found in literature. Shortly, in the MP2 the electrons are oscillating in a cavity in such a manner that they impinge the surface every half-integral $(1 / 2,3 / 2,5 / 2, \ldots)$ number of the rf period. In the MP1, the electrons hit the surface after an integer number of periods. This number of incomplete periods in the case of MP2 or complete periods in the case of MP1 is called the order of MP. All the time between collisions with walls the electrons are subjected to the forces from both electric and magnetic fields. After the collision, depending on the energy of the electron at this moment, some quantity of secondary electrons is released from the wall. If this quantity is more than one in average and conditions for the newly appeared electrons are appropriate, they can be again accelerated in the inner space of the cavity and newly hit out secondary electrons. So, the number of electrons can increase exponentially and lead to a significant loss of the energy of the cavity. The multipacting electrons can increase the temperature of the wall that can be registered by the thermometers installed on the outer surface of the cavity [1].

\footnotetext{
*vs65@cornell.edu
}

Published by the American Physical Society under the terms of the Creative Commons Attribution 3.0 License. Further distribution of this work must maintain attribution to the author(s) and the published article's title, journal citation, and DOI.
PACS numbers: 52.80.Pi, 79.20.Hx, 02.60.-x, 85.25.-j

Geometry from [1] will be used for illustration of further consideration because this paper gives a very detailed and precise description of MP. This paper presents also data of simulation. The software for this simulation is described in another paper of the same time [2]. It was possibly the first serious program for MP simulation. It included a Monte Carlo estimation of the number and momentum of any secondary particles, secondary and backscattered electrons, production of photons, and photoelectric and Compton effects. One of the conclusions in this paper was the following: the "electron avalanche multiplication effect ... is nonresonant in nature." One can see a contradiction of this statement with the results from [1] where it is shown that "the multipacting is limited to discrete field levels." The question arises: if these field levels are not resonances why are they discrete? In the following, we will try to answer this question.

Of course, the simulations reveal the necessary conditions for the MP. But the kind of the MP is defined a posteriori, as a result of the simulation. Understanding of the conditions of the existence of this or another kind of MP can give a clue to prevent it.

The commonly used superconducting cavity shape is a result of evolution from a pillbox rf cavity with the beam pipes added and rounded wall corners-to decrease the peak electric field-to a shape consisting of elliptic arcs to prevent multipacting [3]. A cavity with a profile line consisting of an elliptic arc as the equator region and of an elliptic arc as the iris region, with these two arcs connected by a tangent straight segment, is usually called an elliptic cavity.

The most radical way to avoid MP is to decrease the secondary emission yield (SEY) below unity. For this goal the surface can be covered by materials having SEY $<1$, for example a soot or TiN. Unfortunately, this cannot be done for superconducting cavities. Additional magnetic or electric, static or rf, fields known as remedies against MP are also unacceptable in SC cavities. So, the choice of the shape stable against MP appears the only way to avoid it. 
As it is said above, the shape consisting of elliptic arcs helps to avoid MP on the surface remote from the axis and alleviate the problem near the cavity equator where the MP though happens but can be easily processed. The principle of the electric field minimum for MP proposed in [4] explains why the equator stays the place where the MP2 occurs and helps avoid it in the transitions between cavities and beam pipes. In the present paper, it will be shown that this principle holds also for the MP1.

An analysis of the two-point MP near the cavity equator was presented recently in detail [5]. Now we will concentrate our attention on the MP1 in the SC rf cavities, analyze some existing experimental data, and compare these two kinds of the rf discharges.

Because of small sizes of the electron trajectories it is possible to expand fields near the MP site into Taylor series and solve the differential equations of motion in the nearest vicinity of this site. Parameters of these equations become the figures of merit of the MP and the control of them gives a clue to prevent MP.

\section{FIELDS AND EQUATIONS OF MOTION IN A KNOWN GEOMETRY WITH ONE-POINT MP}

Description of a MP1 can be found, for example, in [1] or in [6]. The last paper deals with rather complicated geometry of a so-called muffin-tin cavity and the other one geometry is closer to the contemporary accelerating cavities. Trajectories of the multipacting electrons illustrated in both papers are very similar, and we will choose for the analysis the cavity from the first paper which has the shape closer to the elliptic shapes.

Though not all the dimensions of the cavity are presented in the paper, we can approximately define them from the given drawing and frequency. This cavity is not exactly an elliptic one: it has a flat equatorial region. The rounding between this region and the tilted wall has a radius $R=4.7 \mathrm{~mm}$, Fig. 1. We defined from this figure with some uncertainty the equatorial radius $R_{\text {eq }}=43.6 \mathrm{~mm}$ and the wall slope angle $\alpha=85^{\circ}$. This flatness near the equator which is absent in the elliptical cavities appears responsible for MP.

The fields in the cavity were calculated with SLANS [7]. This software gives the best accuracy between the 2D programs but even in this case by reasons explained later we needed to use a very dense mesh $(180 \times 100)$. It is also shown in Fig. 1.

As it is shown in Fig. 1, MP occurs near the place where the flat part of the cavity surface changes to the rounded region. The electric surface field for different values of the radius $R$ is shown in Fig. 2, fields in the volume near this place for $R=4.7 \mathrm{~mm}$ are presented in Fig. 3. One can see that in the vicinity of the MP spot with the dimensions $2 \times 2 \mathrm{~mm}$ (upper estimation of the size of the particle orbit) the radial component of the electric field $E_{r}$ deviates from the average value by $\pm 16 \%$, the magnetic field $H$ is constant with accuracy of $\pm 2 \%$, and the longitudinal electric field $E_{z}$ is linear with the distance from the surface. The difference in the $E_{r}$ between the equator and this spot at some distance from the symmetry plane consists in the following: $E_{r}$ is proportional to the distance from the equator and changes its sign when one passes over the equator, but at some distance, if the size of the spot is smaller than this distance, $E_{r}$ varies only slightly. This variation of $E_{r}$ with $z$ can be also accounted but we will first simplify our consideration and do the correction later.

Up to now, and in Figs. 3 and 4, we used a "natural" coordinate system - with the origin at the cavity center, axis $Z$ along the axis of rotation and axis $R$ perpendicular to it. For further consideration we will introduce a Cartesian coordinate system.

So, placing a new system of coordinates with the origin at the MP spot, with axis $x$ parallel to the axis of the cavity and directed from the equator, with axis $y=R_{\mathrm{eq}}-R$ directed from the surface to the axis of the cavity $\left(R_{\text {eq }}\right.$ is the equatorial radius and $R$ is distance from the cavity
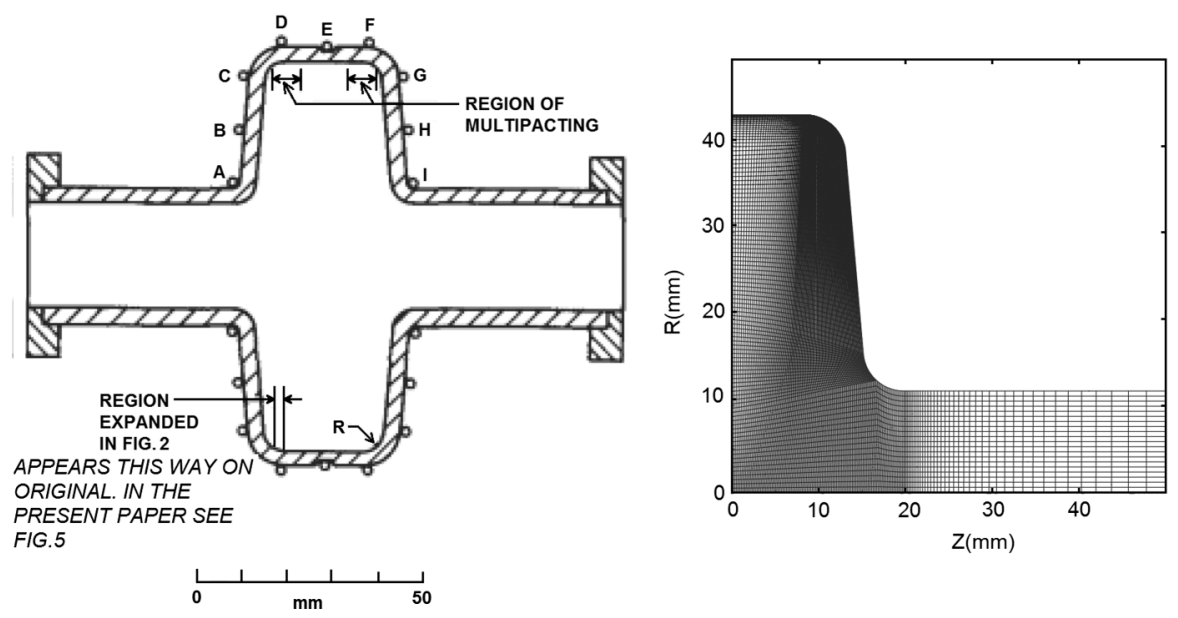

FIG. 1. Schematic drawing of the S-band cavity from [1] and geometry with mesh for SLANS calculation. 

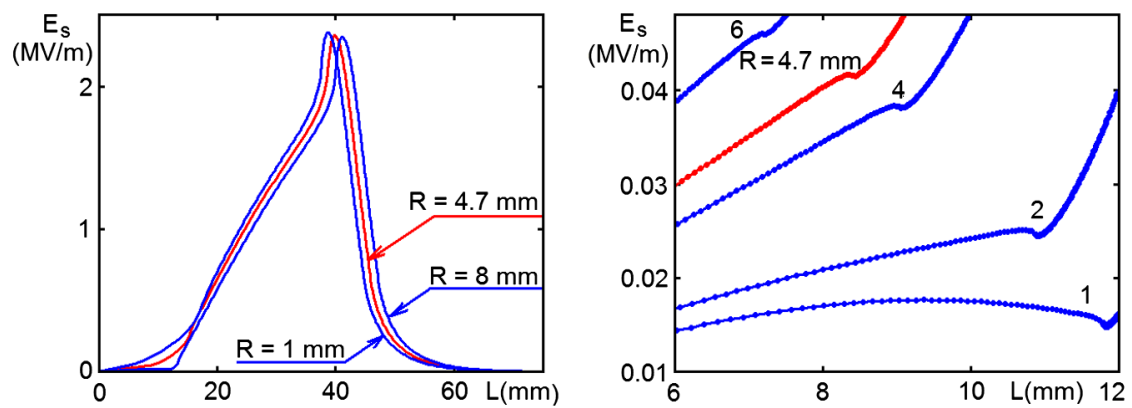

FIG. 2. Surface electric field along the profile line of the cavity. $L$ is distance from the equator. The right figure is not a direct expansion of the left one: more curves are in the right figure to show a shift of the minimum in detail.
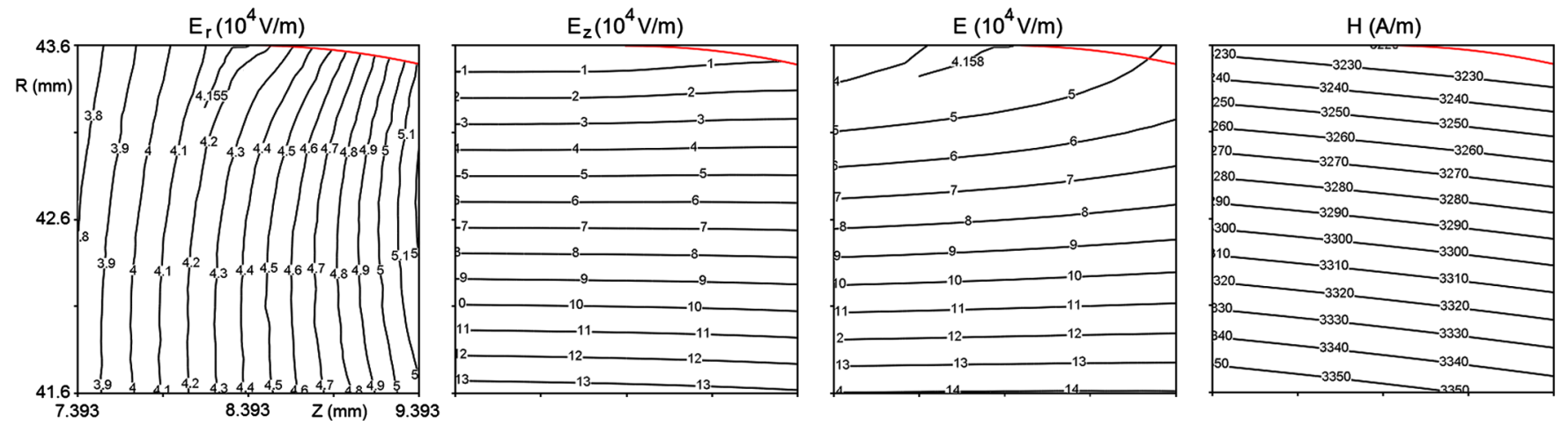

FIG. 3. Electric and magnetic fields near the MP spot of the cavity from Fig. 1. Radius of rounding of the outer cylindrical wall $R=4.7 \mathrm{~mm}$. Total energy of the field in the cavity is $1 \mathrm{~mJ}$ in all the calculations.
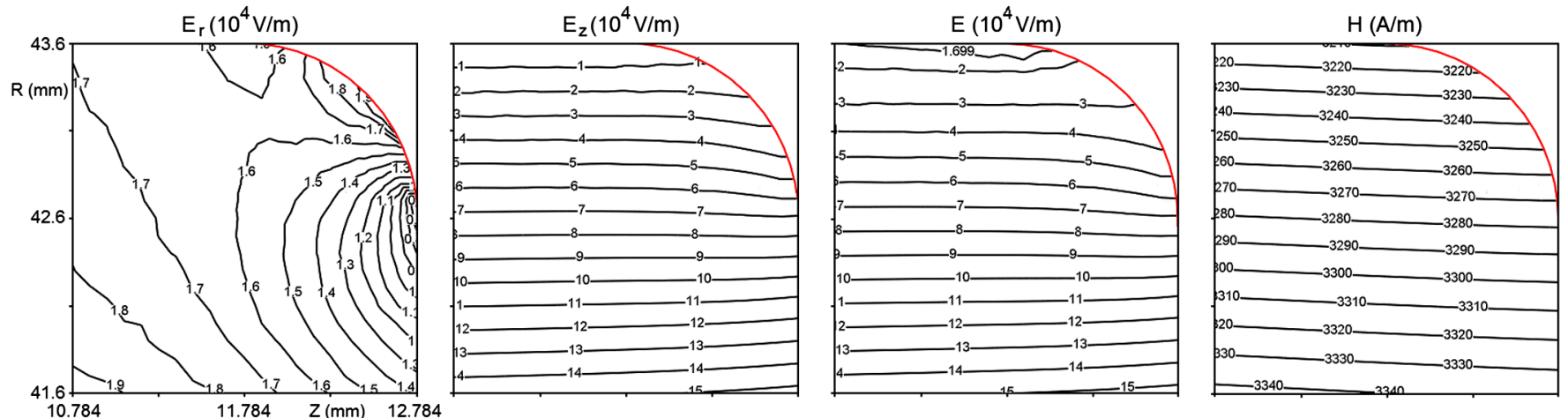

FIG. 4. Electric and magnetic fields near the rounded corner with $R=1 \mathrm{~mm}$.

axis), and with an axis $z$-supplementing this right-hand system of coordinates, we can present the electric and magnetic fields in the form

$$
E_{x}=-\alpha y \sin \theta, \quad E_{y}=E_{0} \sin \theta, \quad B_{z}=B_{0} \cos \theta .
$$

Here $\theta=\omega t$.

From the Maxwell's equation

$$
\oint_{l} \vec{E} d \vec{l}=-\frac{\partial B}{\partial t} \cdot S
$$

for any small contour around the MP spot with length $l$ and surface area $S$ we can find

$$
\alpha=\omega B_{0}
$$

Now we can write the equations of motion taking into account these three components of fields:

$$
e\left(E_{x}+\dot{y} B_{z}\right)=m \ddot{x}, \quad e\left(E_{y}-\dot{x} B_{z}\right)=m \ddot{y} .
$$

$e$ and $m$ are the charge and mass of the electron, the charge is taken positive to simplify writing. Dots designate derivatives with respect to time. Replacing derivatives with respect to time by derivatives with respect to the phase angle $\theta$ (designated by primes), so that 


$$
\dot{x}=\omega x^{\prime}, \quad \ddot{x}=\omega^{2} x^{\prime \prime}, \quad \text { and so on, }
$$

one can obtain the equations of motion in a normalized form:

$$
\begin{aligned}
& x^{\prime \prime}=M\left(-y \sin \theta+y^{\prime} \cos \theta\right), \\
& y^{\prime \prime}=M\left(q \sin \theta-x^{\prime} \cos \theta\right) .
\end{aligned}
$$

Let us call $M=e B_{0} / m \omega$ a magnetic parameter of the motion, and $q=E_{0} / \omega B_{0}$ can be named the geometrical parameter of MP1 depending on the cavity shape but not on the field amplitudes. $M$ is dimensionless and $q$ has a dimension of the length and for the geometry in Fig. 1 $q=0.605 \mathrm{~mm}$ at the initial point of the rounding ( $Z=8.393, R=43.6 \mathrm{~mm}$, Fig. 3). In what follows, the size of the trajectory appears to be of the order of $q$.

The right part of the first equation in (4) is an exact derivative. So, we can integrate this equation and substitute $x^{\prime}$ into the second one. After this we will have

$x^{\prime}=M y \cos \theta, \quad y^{\prime \prime}+M^{2} y \cos ^{2} \theta-M q \sin \theta=0$.

The first equation in (5) means that the $x$ component of the velocity is always zero when the electron impacts the surface if it had been started normally to the surface. Therein lies a difference with the two-point MP where a considerable angle can be between the normal to the surface and the vector of velocity of the impinging electrons that leads to enhancement of the SEY.

\section{COMPARISON OF ANALYTICAL CALCULATIONS WITH SIMULATIONS AND EXPERIMENT}

In spite of significant simplifications of the equations of motion and an approximate knowledge of the geometry, the orbits obtained solving Eq. (4) are very similar to the orbits in the cited paper [1], Fig. 5.

There are also presented in this paper the axial electric fields $E_{a}$ at which the multipacting intensity was observed to reach a maximum. Of course, in reality there are MP bands, not narrow lines, for each order where the MP
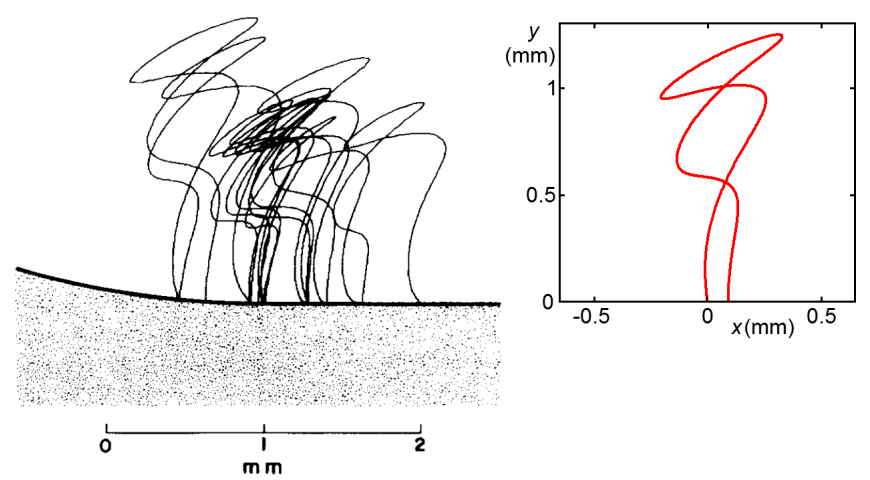

FIG. 5. Example of electron orbits from [1] for third-order MP and a solution of Eq. (4) for the geometry from Fig. 1. exists. These bands were calculated using Eq. (4). Borders of these bands are defined by the condition of stability. This condition is satisfied when an electron having an initial phase deviation from an equilibrium returns to the surface with the phase deviation reduced. An analogous condition should be used for the coordinate on this surface, in a similar way as it was done in [5], but it appeared that no space stability for MP1 was found: the particles always shift to the equator after each flight.

In the solution of Eq. (4) the emission energy of electrons was taken equal to 2 and $4 \mathrm{eV}$. This means that the electrons can be emitted even at a retarding electric field, or at a negative phase. These electrons will be decelerated moving from the surface, and even can start moving back to the surface, and then, if they did not return to the surface, they can be accelerated from the surface again because the phase became positive. The condition of stability can be met for the electrons started at a negative phase. However, if this negative phase is big enough, the electron can touch the same surface before being accelerated and drop out of the resonant process. For the first two orders, the limitation caused by such a return happens earlier than the limitation caused by stability. Boundaries of the MP bands found with Eq. (4) under these conditions are shown in Fig. 6.

These boundaries are found under condition that the secondary emission yield is bigger than 1 for all the energies. In the case of the discussed paper [1], special measures were taken to increase the SEY: the niobium surface was anodized and SEY was enhanced enough to support MP up to sixth order. For different values of impact energy (both lowest and highest) when SEY $=1$, the limits of the MP bands can be easily found with the help of a horizontal line in Fig. 6 corresponding to this energy.

In the discussed paper the field band of the first order is not presented. Results of the simulated MP together with experimental maxima of MP from [1], and our analytical results for the 2-6 orders are shown in Fig. 7. There are also shown the calculated maximal impact energy from the simulations. One can see good agreement between the analytical results and the experiment. The analytical solutions are much closer to the experiment than the simulation

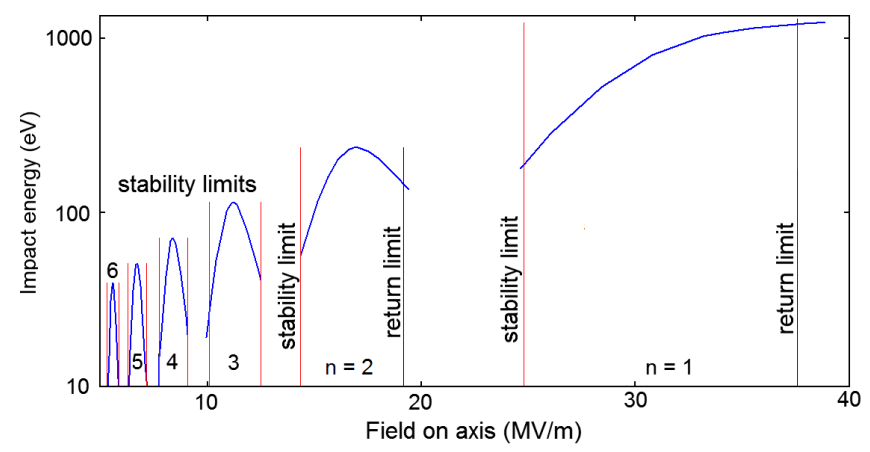

FIG. 6. Analytically found borders of MP1 bands for the geometry [1]. 


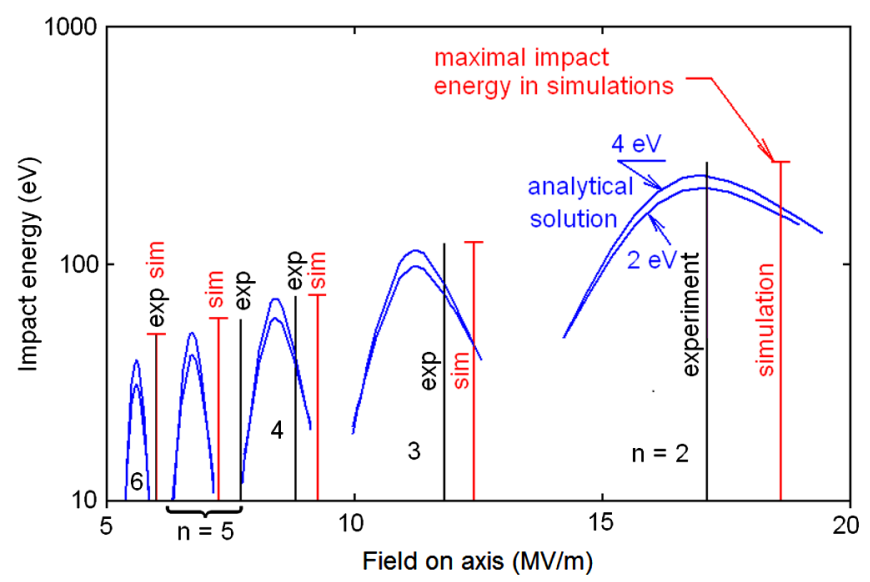

FIG. 7. Comparison of results from [1] and analytical results for 2-6 orders of MP1.

for the second order and have approximately equal deviation from experiment as the simulation for the third and fourth bands. Maximal intensity of simulated MP is at a higher field than in the experiment for all bands but the fifth, and both simulations and experiment coincide for the sixth band. We can suppose that measurements at lowest fields were not so accurate as at the higher fields.

The difference between simulations and analytical solution is regular: analytically found peak fields are always lower than the field found in simulations. A possible explanation of this fact is involving backscattering, bremsstrahlung, photoelectric and Compton effects into simulations. All of these phenomena are more intense at higher fields and so can shift the peaks to higher values. However, the analytical calculation appears not worth the simulations if compared with the experiment that can mean that all these effects have a not so big influence on the position of the bands. These effects define the x-ray energy spectrum produced by electrons impacting with the cavity walls; the analytical calculation cannot predict this spectrum. Maximal intensity of MP apparently occurred at the highest impact energy, this energy was calculated for two values of the emitted electrons: 2 and $4 \mathrm{eV}$. We could not find a reference for the emission energy used in simulations in the cited papers $[1,2]$ though it is mentioned that this

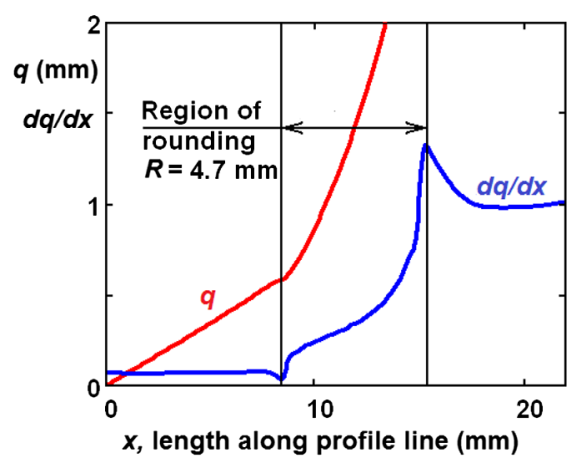

energy is statistically distributed using a Monte Carlo technique. Comparing maxima of impact energies in Fig. 7 for simulations and for analytical solution, one can suppose that the mean energy in simulations is close to $4 \mathrm{eV}$. In the analytical approach no distribution of energies was used. As can be seen from the results, this has only a slight effect on the width of the bands.

\section{INFLUENCE OF CHANGE OF THE SURFACE ELECTRIC FIELD. MULTIPACTOR MAP}

We can take into account changing of the radial component $E_{r}$ shown in Figs. 3 and 4 [or $E_{y}$ in designation of Eq. (1)] along the surface of the cavity. Let us consider as the radial component the surface component of the electric field, $E_{s}$. In this case we can consider also the curved part of the cavity profile line, so that $x$ now can be a coordinate along the "straightened" profile line. This is possible if the particle orbit size is much smaller than the curvature radius. In this case we can change the second equation in (1) to

$$
E_{y}=E_{s}(x) \sin \theta
$$

Relationship (2) will change to

$$
\alpha=\omega B_{s}-d E_{s} / d x,
$$

and the normalized equations of motion (4) will take this form:

$$
\begin{aligned}
& x^{\prime \prime}=M\left[(d q / d x-1) y \sin \theta+y^{\prime} \cos \theta\right], \\
& y^{\prime \prime}=M\left[q(x) \sin \theta-x^{\prime} \cos \theta\right] .
\end{aligned}
$$

Now, $q(x)=E_{s}(x) / \omega B_{s}(x)$. Behavior of $q(x)$ and $d q / d x$ along the profile line for the geometry from Fig. 1 is shown in Fig. 8 for $R=4.7 \mathrm{~mm}$ and $R=0.38 \mathrm{~mm}$. The origin of the coordinate system is placed at the equator.

Practically, $q(x)$ is proportional to the surface field, Fig. 2, because $B_{s}(x)$ only weakly changes along the profile line. $d q / d x$ at the initial point of the rounding is close to zero or even negative as can be seen from Figs. 8 and 2 . Analytical results presented in Figs. 6 and 7 can be generalized if instead of field on axis we will use the magnetic

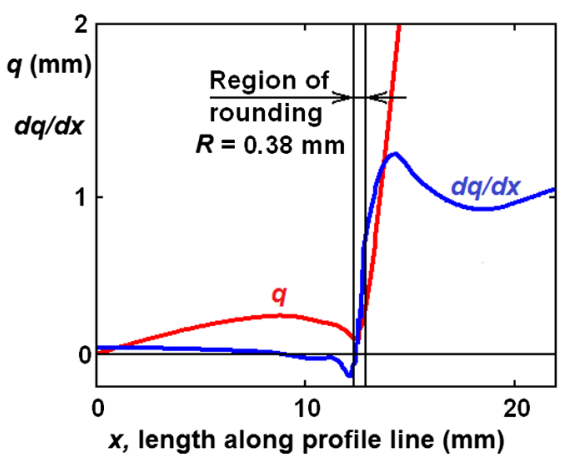

FIG. 8. $q(x)$ and $d q / d x$ for the corner rounding of 4.7 and $1 \mathrm{~mm}$, see geometry in Fig. $1 . x=0$ corresponds to the cavity equator. 


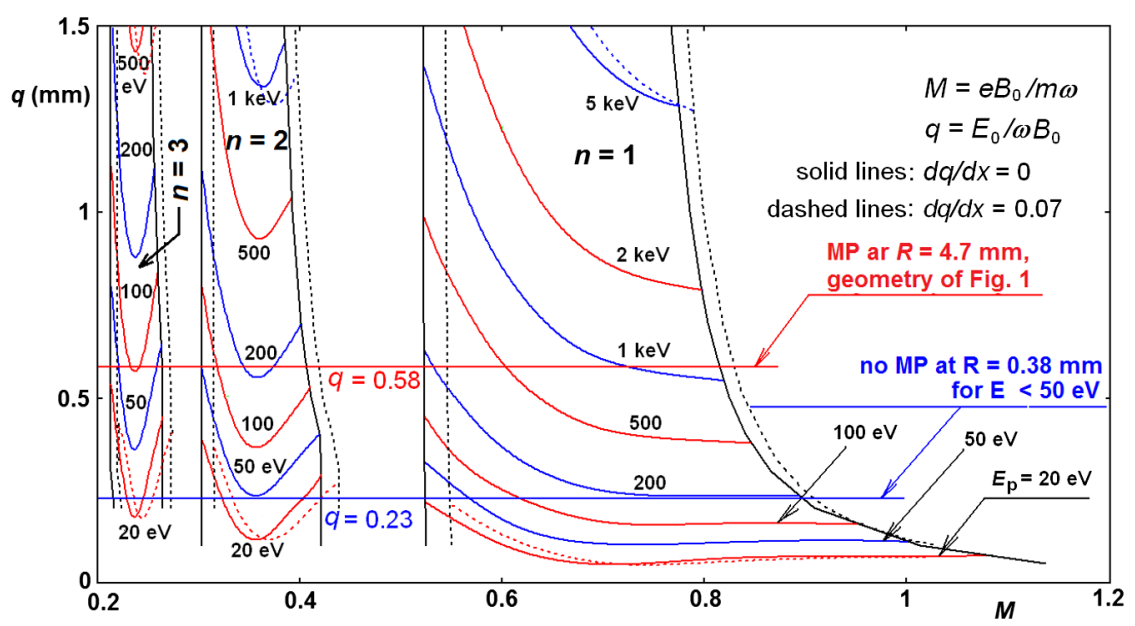

FIG. 9. First three zones of MP1 with $d q / d x=0$ and $d q / d x=0.07$. Emission energy of secondary electrons $E_{s}=4 \mathrm{eV}, E_{p}$ is the impact energy of primary electrons.

parameter $M$ for the surface magnetic field at the equator corresponding to the axis electric field. As another coordinate of this MP map we will introduce the geometrical parameter $q$. The map is presented in Fig. 9.

In the experiment described in [1], five zones of MP were observed (with the order $n$ from 2 to 6 ) with the rounding radius of $R=4.7 \mathrm{~mm}$, the lowest impact energy was $50 \mathrm{eV}$. No multipacting occurred when this radius was changed to $0.38 \mathrm{~mm}$. We can see in Fig. 9 that the maximal energy of multipacting electrons for the second and third zones ( $n=2$ and 3 ), and obviously for higher zones, is below $50 \mathrm{eV}$ if the rounding radius is $R=0.38 \mathrm{~mm}$. The field in the experiment was not high enough to excite the first-order MP: the maximal axial field value was below $20 \mathrm{MV} / \mathrm{m}$ that corresponds to $M=0.42$. So, this generalized description, in terms of geometric $(q)$ and field parameters $(M)$, is also in a full agreement with the experiment.

\section{PHASE AND SPACE STABILITY: TRAVELING MULTIPACTOR}

In the calculation of the zone boundaries (see Figs. 6 and 9), the condition of stability or the condition of return was used, as is described above. After each flight, the particles fall on the surface at a new place shifting from the point of start. This is a difference between MP1 and MP2 where stability in both, phase and space, was found [5].

In Fig. 10, the coordinates of the impact points are shown for the electrons started at the point of beginning of the curvature with $R=4.7 \mathrm{~mm}$. In this consideration we will trace the motion of one particle, so as it has a SEY $=1$ and after the strike on the surface a new one particle appears at the same place with energy of $4 \mathrm{eV}$ and the velocity directed normally to the surface. The lower curve is calculated for $M=0.35$, and the initial $q=0.58 \mathrm{~mm}$ (see Fig. 9), with $d q / d x=0$. Calculation results have shown that the particle can make about 30 impacts with $E_{p}>50 \mathrm{eV}$, so that a big number of secondary electrons can be produced. The upper curve is calculated for $M=0.379$, the same initial $q$, but with a realistic value of $d q / d x=0.07$. The values of $M$ are chosen so that the stable phase angle is close to 0 . The value of $q$ was recalculated for the lower curve after each impact according to the coordinate shift (Fig. 8, left). It is seen that

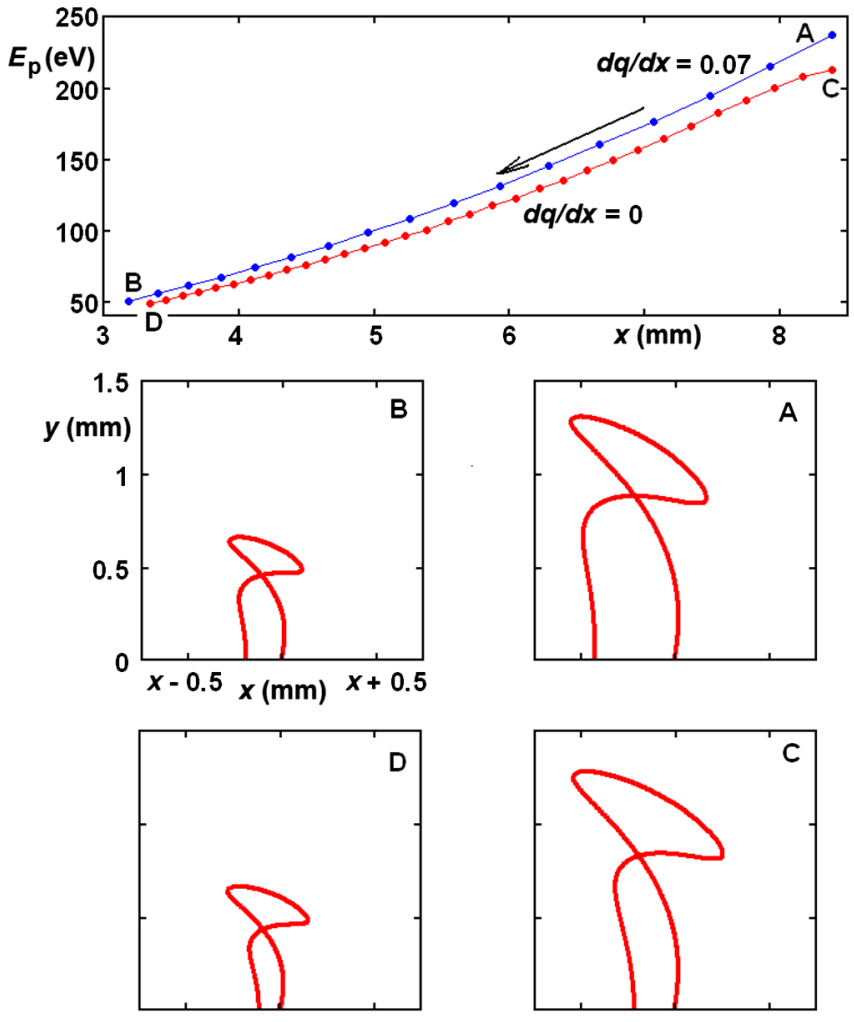

FIG. 10. Coordinates of the impact points for the electrons started at the curvature beginning point. Influence of the nonzero $d q / d x$. Trajectories in the extreme points $\mathrm{A}, \mathrm{B}, \mathrm{C}$, and $\mathrm{D}$ are shown in the same scale. 


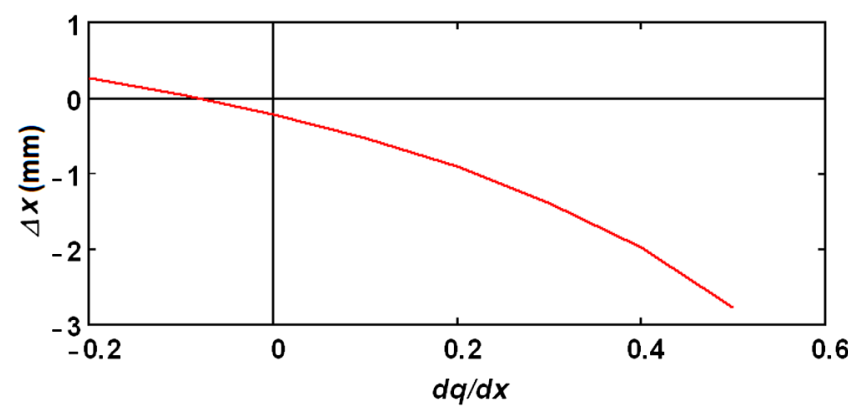

FIG. 11. Dependence of shift of the impact point after each flight on the derivative $d q / d x$.

the account of the derivative in the equations (8) significantly increases the width of the trajectories of the particles though the boundaries of the zones are only slightly shifted (Fig. 9). Nevertheless, this number of impacts, when the electrons are in the region with SEY $>1$, is big enough and the MP can exist. The phase detuning after each flight is very small, less than $0.02^{\circ}$ in all the cases, so that phase stability is conserved.

The measure of the phase stability, $\lambda=\partial \theta_{2} / \partial \theta_{1}$, is changing from 0.135 to 0.287 in the case of $d q / d x=0$, and from 0.134 to 0.280 in the case of $d q / d x=0.07$. Here, $\theta_{1}$ and $\theta_{2}$ are the phases of start and impact, respectively. The condition $|\lambda|<1$ means that a random phase deviation from the equilibrium phase at the start point becomes smaller at the point of impact which is the next start point.
The requirement $|\lambda|=1$ defines the boundaries of the zones, except of the cases of returning particles in the decelerating field. We can conclude that the one-point multipactor is resonant from the viewpoint of phase but the point of impact shifts all the time in the direction to the equator. So, we can name this phenomenon "a traveling multipactor".

In the pictures of the paper [2] this ordered motion is not seen due to many details taken into account: distributed start velocities, reflected electrons, and simply because too many trajectories are simultaneously shown. These details of the distributed velocities though reflecting the real situation work like a noise hiding the main features of the phenomenon. So, the MP1 is resonant in nature but this is only phase resonance, the point of MP travels along the profile line to the equator.

The point of the curvature beginning has low $d q / d x$ and the MP travels slowly near this point. In the case $d q / d x<0$ (Figs. 4 and 8) the reverse motion is possible but the value of $q$ is too low and hence the energy of impact is too low and $\mathrm{SEY}<1$.

The length of the segment with $d q / d x \leq 0$ in Fig. 8 is rather short and the particle cannot make many "steps" in this region. This is why the example presented in Fig. 10 with $d q / d x=0$ is artificial: it should show only that for different $q$, if there is "a point of rest"-with a small $d q / d x$, like at the beginning of the rounding-the probability of MP increases. In more detail the influence of the
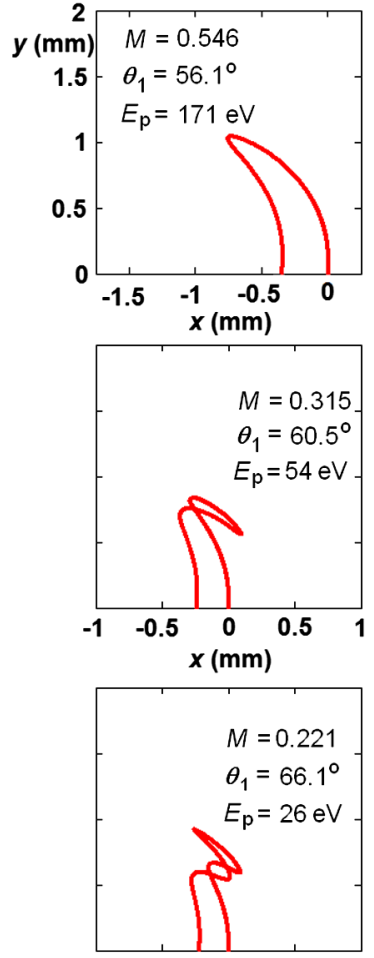
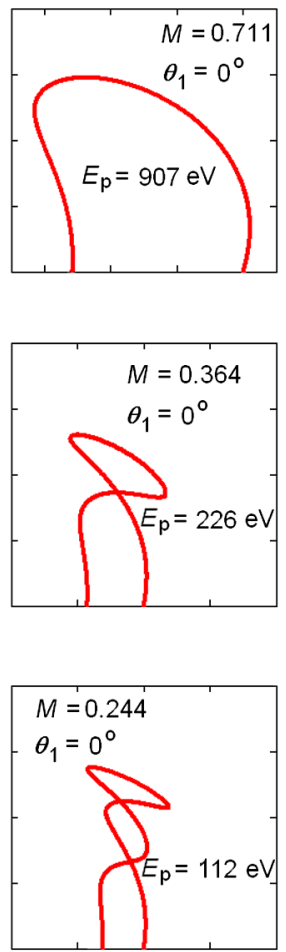
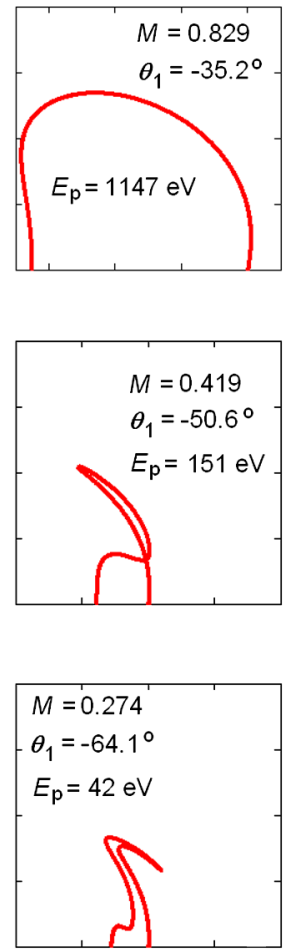

FIG. 12. Examples of orbits for the first three zones of MP1. The upper row is for $n=1$. Compare values of $M$ at $q=0.58$ with Fig. 9. 
value of $d q / d x$ on the shift in each flight is shown in Fig. 11. Here the phase angle is also chosen zero, within the 2nd multipactor zone. The value of $q$ is taken $0.58 \mathrm{~mm}$. One can see that the number of steps to the place where $E_{p}=50 \mathrm{eV}$ made by the electron, each step from emission to the impact, will decrease fast if the value of $d q / d x$ becomes bigger. Besides, for bigger $d q / d x$ the phase shift between consecutive flights becomes different and the particle goes out of the phase resonance. Small enough derivatives can be in the cavities like in Fig. 1: with a flat equator. The elliptic cavities have a big $d q / d x$, for example, for the TESLA cavities this value is 0.29 and for the Cornell ERL cavity it is 0.28 [5], so that MP1 is unlikely in such a geometry. On the other hand, the geometric parameter $p$ introduced in [5] (see also in the next section), is about $p=d q / d x=0.07$ for the cavity of Fig. 2, and MP2 does not happen at its equator, MP2 happens when $p$ is about 0.3 and higher.

Examples of the orbits for $q=0.58, d q / d x=0.07$ at the beginning, in the center, and at the end of the first three zones are shown in Fig. 12. It is seen that the sizes of orbits do not differ too much in spite of different values of fields and different phases of start $\left(\theta_{1}\right)$ : all pictures have the same scale. The energy of impact $\left(E_{p}\right)$ of the primary electrons is also shown. The start of the secondary electrons in a stable motion can occur at different phase angle, not at $\theta_{1}=0$ only. However, for higher zones, starting from the 2nd one, maximal energy and accordingly maximal SEY happens at the angle close to zero that also follows from Fig. 9.

It is worth noting that the sizes of orbits do not scale with the size of the cavity but remain the same because the initial energy of the secondary electrons remains the same: about $2-4 \mathrm{eV}$. This means that the presented approach will work better for bigger cavities of the same shape because the assumption that the sizes of orbits are smaller than the curvature radius will be better satisfied.

\section{COMPARISON OF THE EQUATIONS OF MOTION FOR MP1 AND MP2}

Equations of motion in the case of two-point MP are derived in [5]:

$$
\begin{aligned}
& x^{\prime \prime}=M\left[(1-p) y \sin \theta-y^{\prime} \cos \theta\right], \\
& y^{\prime \prime}=M\left(-p x \sin \theta+x^{\prime} \cos \theta\right) .
\end{aligned}
$$

The geometrical parameter of the two-point MP is defined as $p=\left(d E_{s} / d x\right) / \omega B_{s}$. Difference in signs between (8) and (9) on the right sides means only a shift of the origin of the phase angle $\theta$ by the value of $\pi$. If we take $q=p x$, the sets (8) and (9) coincide, correct to the phase shift by $\pi$. Difference in solutions results from the fact that in the case of MP2 the particle changes the sign of the value of $x$ each time when it flies over the equator and in the case MP1 the particle is at the same side from the equator moving closer to it step by step. On the map of the MP2, only one point corresponds to a given value of the field parameter $M$ because the value of $p$ is defined in one point only, at the equator. In the case of MP1, to each value of $M$ a line corresponds on the map of Fig. 9 because different $q$ 's can be found on the profile line. Particles make big steps along this line at bigger $q$ 's, can slow down at low derivatives $d q / d x$, and, if the number of steps with SEY $>1$ is big enough, the multipactor is on.

Equation (9) is homogeneous; this means that if all the functions - $x, y$, and their derivatives-are multiplied by a constant, the equations keep true. For example, the sizes of the trajectories as well as velocities and accelerations at each point of the trajectory increase $\sqrt{2}$ times if the emission energy is doubled. Conditions of stability will also hold if the emission velocity changes.

This is also true in the case of MP1 if the value of $q(x)$ in (8) is proportional to $x$. Moreover, while in the case of MP2, the changing of initial velocity around the mean value will cause a deviation from the mean trajectory, which will decrease at the next flight due to phase stability, in the case of MP1 the phase will be stable if the velocity changes; only the size of the step to the equator will change.

The value of $p$ in (9) is dimensionless and as it is shown in [5] does not depend on frequency but only on the shape of the cavity. The same is true for the MP1 if $q$ is proportional to $x$. This means that the map of MP1 as well as the map for MP2 in [5] can be used for any frequency.

As follows from the above consideration, if the secondary electrons have a mean value of energy, say $2 \mathrm{eV}$, all the data for $E_{p}$ in Fig. 9 should be taken 2 times smaller.

The angle the electrons impinge the surface can change significantly in the case of MP2, increasing the surface emission yield. This angle is zero for MP1, as follows from (5) for $q=$ const, but it also appears small $\left(<5^{\circ}\right)$ for the discussed model with $d q / d x=0.07$.

\section{DISCUSSION AND CONCLUSIONS}

The past studies of multipacting in superconducting radio frequency cavities have led to the adoption of the elliptical shape for high- $\beta$ resonators. From this point of view, the shape analyzed in the present paper is obsolete because MP1 does not occur in the elliptical cavities. However, many future superconducting rf accelerators demand low- $\beta$ resonators such as quarter-wave, half-wave, and spoke resonators. Different deflecting cavities also present a wide variety of shapes prone to multipacting. Vacuum rf transmission lines, coaxial lines and waveguides, couplers and antennas on the satellites-this is not a full list of devices where this rf discharge occurs. The author hopes that a general approach involving such concepts as geometric and field parameters and a principle of electric field minimum will help in better understanding of this phenomenon. 
An explicit description of the one-point multipactor is presented in this paper, geometrical parameters, or figures of merit, responsible for initiation of the MP defined, and areas of its existence delineated. It was done following the analogous description of the two-point multipactor on the cavity equator [5]. A brief outline of these two cases of MP was also presented recently [8]. Small sizes of trajectories in the MP require a very precise calculation of fields for simulations. On the other hand, due to these small sizes, fields can be presented as the Taylor expansions and trajectories can be found solving ordinary differential equations of motion. Conditions of the phase stability define the boundaries of the MP zones. It is shown that the MP discharge can be described in terms of two parameters: the geometrical and the field parameter. The parameter $p$ on the equator or the parameter $q$ along the profile line of the cavity and the value of the field parameter $M$ at these points define the conditions when one- or two-point MP will occur. In any responsible design of an rf cavity one calculates fields on its surface. These fields can be immediately used for calculations of the geometric and field parameters from which a conclusion about possibility of MP can be done. A comparison with some experimental data shows that this approach gives a very close result to the simulations and experimental data. The maps of multi- pacting give a way to guess a possibility of this or the other kind of multipactor without time consuming simulations.

\section{ACKNOWLEDGMENTS}

This work was supported by NSF Award No. DMR0807731.

[1] C.M. Lyneis, H.A. Schwettman, and J.P. Turneaure, Appl. Phys. Lett. 31, 8 (1977).

[2] I. Ben-Zvi, J.F. Crawford and J.P. Turneaure, in Proceedings of the 5th Particle Accelerator Conference, San Francisco, CA, 1973 [IEEE Trans. Nucl. Sci. 20, 54 (1973).

[3] H. Padamsee et al., RF Superconductivity for Accelerators (John Wiley \& Sons, New York, 1998).

[4] S. Belomestnykh and V. Shemelin, Nucl. Instrum. Methods Phys. Res., Sect. A 595, 293 (2008).

[5] V. Shemelin, Phys. Rev. ST Accel. Beams 16, 012002 (2013).

[6] H. Padamsee and A. Joshi, J. Appl. Phys. 50, 1112 (1979).

[7] http://www.euclidtechlabs.com/SLANS/slans.php.

[8] V. Shemelin, in Proceedings of the 4th International Particle Accelerator Conference, Shanghai, China, 2013, p. 2456. 